\title{
Identification of Spectrum Sharing Opportunities for a Finite Field Secondary Network through an Exact Outage Expression under Rayleigh Fading
}

\author{
Arshdeep S. Kahlon, Sebastian S. Szyszkowicz, Shalini Periyalwar, Halim Yanikomeroglu \\ Department of Systems and Computer Engineering, Carleton University, Ottawa, Ontario, Canada \\ E-mail: \{akahlon, sz, shalinip, halim\}@ sce.carleton.ca
}

\begin{abstract}
A closed-form expression for the outage probability at a receiver in the primary network due to a uniform annular sector distribution of secondary transmitters is derived in a Rayleigh fading environment. This may include an exclusion region, and a finite or infinite outer radius. Conversely, under a given constraint on the outage probability, spectrum sharing opportunities are analyzed with respect to the field size and are shown to be significantly dependent on the exclusion region. Conditions are obtained for which an infinite field size assumption can or cannot lead to the loss of spectrum sharing opportunity. Additionally, the spectrum sharing gains (the maximum average number of secondary transmitters) are derived for the secondary network for different spatial deployments. It is worth mentioning that other than networks sharing spectrum, the derived results are applicable to any set of interferers from the same network or other networks around any receiver of study.

Index Terms-Spectrum sharing, outage probability, secondary/cognitive network, exclusion region, stochastic geometry.
\end{abstract}

\section{INTRODUCTION}

Due to spectrum scarcity, various spectrum sharing scenarios have been proposed in the literature. The principal performance metric in such studies at the physical layer is the outage probability at the receiver of the incumbent primary network due to the secondary network. A typical scenario is one where a secondary network shares the spectrum of a primary user in the underlay scheme [1]. The network operators would like to get insights into the outage probability at a primary receiver due the deployment of a secondary network. Conversely, there is an opportunity for the secondary network to share the spectrum if it satisfies the outage probability constraint at the primary receiver. It is also important to know what spectrum sharing gains (notably, the maximum number of secondary transmitters) can be achieved given the outage probability constraint by the primary user.

Most of the previous works focused on scenarios where the primary user spectrum is accessed by a single user, as given in the references in [1]. The number of possible network configurations increases dramatically with the increase in the number of nodes. Hence, a deterministic approach used for a few nodes is highly challenging to scale to large networks (as in femtocells and ad hoc networks) where the number, as well as the positions, of the nodes are random. Often, the secondary transmitters are modeled as the points of a homogeneous Poisson point process (HPPP) on a two-dimensional plane [2].
A significant amount of literature focuses on scenarios where the secondary transmitters are deployed around a typical primary receiver of study. Sometimes, an exclusion region around this receiver is assumed [3]-[6]. Several works [4], [6][8] perform curve fitting of the interference distribution, using the moment (or cumulant) matching approach or numerical methods; in some of these cases, only the asymptotically small outage probability conditions are studied. Another approach to study the tradeoff between the secondary transmitter density and outage probability in low outage probability regimes is to consider only the dominant interferer [9]. The maximum interferer density is derived in [10] for Rayleigh fading channels under an outage probability constraint specified by the primary receiver with no exclusion region around it.

In all of these works, the secondary network is assumed to be distributed over an infinite field for analytical simplicity, which may not be an accurate model for a finite field secondary network. The asymptotic results obtained in these cases are too pessimistic in the sense that the interference is overestimated, and spectrum sharing opportunities could be lost. The effect of the interferers' field size is studied in [5], where the conditions for an infinite field to be a valid approximation to a finite field are explored through bounds on the outage probability. In [11], the dominant regions that dictate spectrum sharing opportunities are studied using the cumulant matching approach. Outage probability bounds at the typical primary receiver are obtained in [3], with exclusion regions around both the primary transmitter and primary receiver for the finite outer region. However, tight bounds are finally obtained by assuming an infinite outer region. Outside the context of spectrum sharing, integral expressions for outage probability at any receiver in a single ad hoc network are given in [12], with the interfering transmitters distributed over an annular region. A bound for the outage probability is derived in closed form, but only for an infinite outer radius.

In this paper, we derive a closed-form expression for the outage probability when the secondary network is distributed according to an HPPP over an annular sector region (which may include an exclusion region, and a finite or infinite outer radius) around the primary receiver. Conversely, we derive the maximum allowable density of the secondary network, given a constraint on the outage probability by a primary victim receiver. Using these results, we analyze the conditions under 
which an infinite field size assumption can lead to a loss of spectrum sharing opportunity. Furthermore, we derive the spectrum sharing gains (expressed in terms of the maximum average number of secondary transmitters) as a function of the spatial deployment under the given outage probability constraint. We confirm the analytical results through Monte Carlo simulations.

The remainder of this paper is organized as follows. Section II provides a general system model of the problem we intend to solve, and gives the starting point for the analysis. In Section III, the outage probability expression in the case of a finite sector field of the secondary network is obtained and spectrum sharing opportunities are analyzed. In Section IV, the analytical results are validated against simulations. Finally, concluding remarks are presented in Section V.

\section{Problem Statement}

We consider a primary receiver (that can be assumed a typical primary receiver within the primary network) at the origin where the outage probability is studied. For a given mean received power $\rho_{\mathrm{r}}$, the signal power received at this receiver node is given by

$$
S=\gamma_{0} \rho_{\mathrm{r}},
$$

where $\gamma_{0}$ is an exponentially distributed random variable with unit mean, representing Rayleigh fading on the desired signal link.

Let $\varepsilon$ be the outage probability at the primary receiver node due to the interference power $I$ coming from the secondary network and to the background noise power $\eta$; it is given by

$$
\mathcal{P}\left(\frac{S}{I+\eta}<\beta\right)=\varepsilon,
$$

where $\beta$ is the signal-to-interference-and-noise power ratio (SINR) threshold.

\section{Outage Analysis}

Proceeding similarly to [6], we say that the secondary transmitters are distributed around the primary receiver as the points of a point process $\Pi=\left\{X_{i}\right\}$ over $S \subseteq \mathbb{R}^{2}$, as shown in Fig. 1 . Corresponding to $\Pi$, we define $\Pi^{\prime}=\left\{\left(X_{i}, \gamma_{i}\right) \mid X_{i} \in \Pi\right\}$ over the product space $S \times \mathbb{R}^{+}$, where $\gamma_{i}$ is the mark associated with the interferer at $X_{i}$, representing fading on the channel between this interferer and the typical primary receiver. The marks are distributed identically and independently for every $i$, and independently of $X_{i}$.

In the case when $\Pi$ is a Poisson point process (PPP), then from the Marking Theorem [13], because the marks $\gamma_{i}$ are independent, it follows that $\Pi^{\prime}$ is also a PPP. We then apply Campbell's theorem [13] for a marked PPP to $\Pi^{\prime}$ in order to obtain the Laplace transform of the distribution of $I$ at the primary receiver:

$$
\mathcal{L}_{I}(s)=\exp \left\{\int_{S} \mathbb{E}\left(e^{-s \gamma \rho(r, \theta) g(r)}-1\right) \lambda(r, \theta) r d r d \theta\right\},
$$

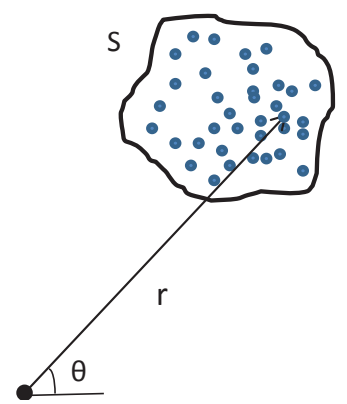

Fig. 1. Secondary network transmitters distributed as a PPP over some general region around the primary receiver.

where $\lambda(r, \theta)$ is the density of secondary transmitters in $\Pi$ at $(r, \theta), \rho(r, \theta)$ is the power transmitted by the secondary transmitter at $(r, \theta), g(r)=r^{-\alpha}$ is the path loss function, and $\gamma$ is an exponentially distributed random variable with unit mean, i.e., with the same distribution as the marks $\gamma_{i}$.

We calculate the outage probability $\varepsilon$ at the primary receiver due to the interference coming from the secondary network and the background noise. The outage probability can be expressed as the product of the Laplace transform of the interference $I$ and noise $\eta$ distribution [14]. We provide a brief derivation of this result as follows. Using (1), we can express (2) as

$$
\begin{aligned}
& \mathbb{E}\left(\mathcal{P}\left(\gamma_{0}<\kappa(I+\eta) \mid I, \eta\right)\right)=\varepsilon \\
\Rightarrow & \mathbb{E}\left(e^{-\kappa(I+\eta)}\right)=1-\varepsilon \\
\Rightarrow & \mathcal{L}_{I}(\kappa)=\frac{1-\varepsilon}{\mathcal{L}_{\eta}(\kappa)}
\end{aligned}
$$

where $\kappa=\beta / \rho_{\mathrm{r}}$.

Given a constraint on the outage probability or the maximum outage probability $\varepsilon_{\mathrm{Th}}$ that the primary receiver can tolerate, the secondary network needs to satisfy the constraint

$$
\mathcal{L}_{I}(\kappa) \geq \frac{1-\varepsilon_{\mathrm{Th}}}{\mathcal{L}_{\eta}(\kappa)}
$$

in order to share the spectrum of the primary user.

We may rewrite (3) to obtain

$$
\mathcal{L}_{I}(s)=\exp \left\{\int_{S}\left(\frac{-s \rho(r, \theta)}{s \rho(r, \theta)+r^{\alpha}}\right) \lambda(r, \theta) r d r d \theta\right\} .
$$

Equations (4) and (6) give the outage probability when the secondary transmitters are distributed as general PPP over a general area $S$. In the case that $\Pi$ is an HPPP, i.e., has constant density $\lambda(r, \theta)=\lambda$, we can write the above equation as

$$
\mathcal{L}_{I}(\kappa)=\exp (-\lambda C)
$$

where

$$
C=\int_{S} \frac{\kappa \rho(r, \theta)}{\kappa \rho(r, \theta)+r^{\alpha}} r d r d \theta
$$

depends on the secondary network only. Using (4) and (7), the outage probability at the primary receiver under a given secondary transmitter density $\lambda$ can be expressed as

$$
\varepsilon=\left(1-\exp (-\lambda C) \mathcal{L}_{\eta}(\kappa)\right) .
$$




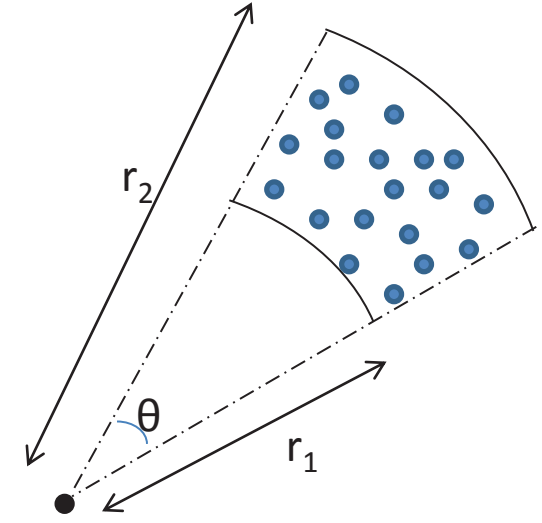

Fig. 2. Secondary network transmitters distributed as an HPPP over a sector around the primary receiver.

Conversely, the maximum density of interferers $\lambda_{\max }$ under the given constraint on the outage probability $\varepsilon_{\mathrm{Th}}$ given by the victim receiver using (5) and (7) is given by

$$
\lambda_{\max }=-\frac{1}{C} \log \left(\frac{1-\varepsilon_{\mathrm{Th}}}{\mathcal{L}_{\eta}(\kappa)}\right) .
$$

Let the secondary network be distributed over a sector $S$, subtending an angle $\theta$ at the primary receiver and between radii $r_{1}$ and $r_{2}$, as shown in Fig. 2. For $\rho(r, \theta)=\rho$, (8) becomes

$$
C=\theta \int_{r_{1}}^{r_{2}} \frac{r d r}{1+r^{\alpha} / \kappa \rho}=\theta\left(p\left(r_{2}\right)-p\left(r_{1}\right)\right),
$$

where

$$
p(r)=\int_{0}^{r} \frac{r d r}{1+r^{\alpha} / \kappa \rho} .
$$

Substituting $x=r^{\alpha}, \beta=1 / \kappa_{1} \rho, \nu=n=1, \mu=2 / \alpha$ in (3.194.1) and (3.194.4) of [15] gives:

$$
p(r)= \begin{cases}\frac{1}{2} r_{2}^{2} F_{1}\left(1, \frac{2}{\alpha} ; 1+\frac{2}{\alpha} ; \frac{-r^{\alpha}}{\kappa \rho}\right), & 0<r<\infty \\ 0, & r=0 \\ \frac{\pi(\kappa \rho)^{2 / \alpha}}{\alpha \sin (2 \pi / \alpha)}, & r \rightarrow \infty,\end{cases}
$$

where ${ }_{2} F_{1}$ is the Gauss-hypergeometric function [15].

It should be noted that the following different geometries are covered by (11):

- $\Pi$ is an HPPP over an infinite area around the primary receiver, i.e., $\theta=2 \pi, r_{1}=0, r_{2} \rightarrow \infty$, [10].

- $\Pi$ is an HPPP over an infinite area around the primary receiver with an exclusion region, i.e., $\theta=2 \pi, r_{1}>$ $0, r_{2} \rightarrow \infty$, [4], [12].

- $\Pi$ is an HPPP over a finite area around the primary receiver with or without an exclusion region, i.e., $\theta=$ $2 \pi, 0 \leq r_{1}<r_{2}<\infty$, [5].

\section{A. Effect of Field Size on the Spectrum Sharing Opportunity}

There is a spectrum sharing opportunity for the secondary network if the probability of outage caused by the secondary network is below some threshold outage probability $\varepsilon_{\mathrm{Th}}$ specified by the primary receiver. Using (5), (7) and (11), we can write

$$
p\left(r_{2}\right)-p\left(r_{1}\right) \leq-\frac{1}{\lambda \theta} \log \left(\frac{1-\varepsilon_{\mathrm{Th}}}{\mathcal{L}_{\eta}(\kappa)}\right) .
$$

A set of triplets $\left(r_{1}, r_{2}, \theta\right)$ that satisfies the above constraint gives the feasible field of the secondary network under the constraint on the outage probability $\varepsilon_{\mathrm{Th}}$ specified by the primary user.

From (12), it can be easily shown that $p(r)$ is a strictly increasing function of $r$ for $r>0$. If the secondary network is distributed over a finite region between $r_{2}^{*}$ and $r_{1}^{*}$, it is possible that

$$
p\left(r_{2}^{*}\right)-p\left(r_{1}^{*}\right) \leq-\frac{1}{\lambda \theta} \log \left(\frac{1-\varepsilon_{\mathrm{Th}}}{\mathcal{L}_{\eta}(\kappa)}\right)<p(\infty)-p\left(r_{1}^{*}\right) .
$$

Therefore, the assumption of an infinite field size, when the secondary network is actually distributed over a finite area, can lead to the loss of the spectrum sharing opportunity, as supported by results in [5].

B. Convergence of Outage Probability to a Value Less than 1 with respect to Field Size

Let us write $\varepsilon\left(r_{2}\right)$ to be the outage probability $\varepsilon$ as a function of the outer field radius $r_{2}$. We find the limit of $\varepsilon\left(r_{2}\right)$ as $r_{2} \rightarrow \infty$ to be

$$
\lim _{r_{2} \rightarrow \infty} \varepsilon\left(r_{2}\right)=1-\exp \left(-\lambda \theta\left(\frac{\pi(\kappa \rho)^{2 / \alpha}}{\alpha \sin (2 \pi / \alpha)}-p\left(r_{1}\right)\right)\right) \text {. }
$$

This is in compliance with the previous studies examined in [2], which confirms that the distribution of the aggregate interference power converges as the field size grows infinite, as long as $\alpha>2$.

It is interesting to observe that in certain cases, even when the outer radius is assumed infinite, the outage probability constraint can still be satisfied, as long as the exclusion radius satisfies

$$
p\left(r_{1}\right) \geq \frac{\pi(\kappa \rho)^{2 / \alpha}}{\alpha \sin (2 \pi / \alpha)}+\frac{1}{\lambda \theta} \log \left(1-\frac{\Delta \varepsilon_{1, \mathrm{th}}}{1-\varepsilon_{1}}\right) \geq 0,
$$

which is obtained from (13) and (14).

\section{Spectrum Sharing Gains}

Spectrum sharing gains can be expressed in terms of the average number of transmitting nodes in the secondary network. In order to obtain the maximum spectrum sharing gains, we want to maximize the average number of secondary network transmitters in a given area. The number of nodes in a given sector is a Poisson random variable with mean $N=\frac{1}{2} \lambda \theta\left(r_{2}^{2}-r_{1}^{2}\right)$. The secondary network selects the field size $\left(r_{1}, r_{2}\right)$ and density $\lambda$ to meet the constraint specified by the primary victim receiver.

For a given $r_{1}, r_{2}$, using (10), the maximum average number of transmitters is given by

$$
N_{\max }=\frac{-\log \left(\frac{1-\varepsilon_{\mathrm{Th}}}{\mathcal{L}_{\eta}(\kappa)}\right)\left(r_{2}^{2}-r_{1}^{2}\right)}{2\left(p\left(r_{2}\right)-p\left(r_{1}\right)\right)}
$$




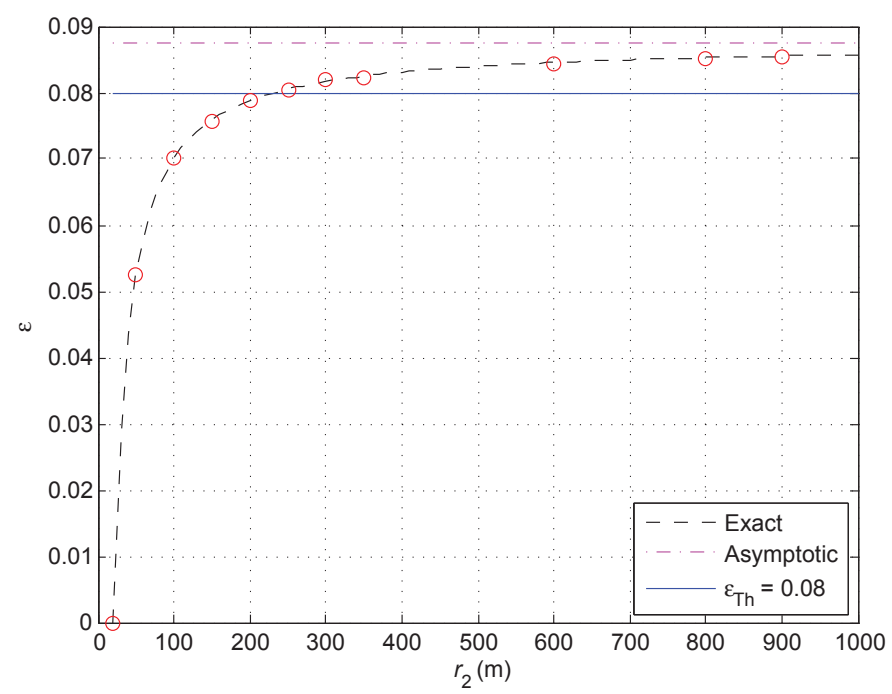

Fig. 3. Outage probability at the primary receiver $\varepsilon$ vs. field outer radius $r_{2}$ of the secondary network. Lines correspond to analysis, while circles represent simulation, with $r_{1}=20 \mathrm{~m}, \lambda=3 \times 10^{-4} / \mathrm{m}^{2}, \theta=2 \pi, \eta=10^{-14}$ $\mathrm{W}, \varepsilon_{\mathrm{Th}}=0.08, \alpha=3, \rho_{\mathrm{r}}=10^{-6} \mathrm{~W}, \beta=10$, and $\rho=10^{-4} \mathrm{~W}$.

Conversely, we can define a contour line on the $\left(r_{1}, r_{2}\right)$ plane for a given $N_{\max }$ that gives the possible regions of deployment.

Also, the secondary network may want to maintain a density $\lambda$ and inner radius $r_{1}$. In that case, we formulate the following optimization problem of maximizing the average number of interfering nodes:

$$
\begin{array}{ll}
\underset{r_{2}}{\operatorname{maximize}} & \frac{1}{2} \lambda \theta\left(r_{2}^{2}-r_{1}^{2}\right) \\
\text { subject to } & p\left(r_{2}\right)-p\left(r_{1}\right) \leq-\frac{1}{\lambda \theta} \log \left(1-\varepsilon_{\mathrm{Th}}\right) .
\end{array}
$$

Both $N$ and $p\left(r_{2}\right)$ are increasing functions of $r_{2}$. Therefore, the optimal outer radius $r_{2}^{*}$ of the field size is given by

$$
p\left(r_{2}^{*}\right)=-\frac{1}{\lambda \theta} \log \left(1-\varepsilon_{\mathrm{Th}}\right)+p\left(r_{1}\right) .
$$

\section{Numerical Results AND Discussions}

In this section, we first validate our derived results using Monte Carlo simulation for the outage probability at the primary receiver, given the field size of the secondary network. We then plot the possible deployments for the spectrum sharing gains of the secondary network. Furthermore, we plot the significant effect of the exclusion region on the outage probability.

For a given secondary network and outage probability constraint at the primary receiver node, we wish to find out if the spectrum sharing opportunity exists, using (14). Additionally, the effect of the field size on the outage probability in (15) is shown in Fig. 3 for $r_{1}=20 \mathrm{~m}, \lambda=3 \times 10^{-4} / \mathrm{m}^{2}$, $\theta=2 \pi, \eta=10^{-14} \mathrm{~W}, \varepsilon_{\mathrm{Th}}=0.08, \alpha=3, \rho_{\mathrm{r}}=10^{-6} \mathrm{~W}, \beta=10$, and $\rho=10^{-4} \mathrm{~W}$. In this case, the spectrum sharing opportunity can be lost by assuming an infinite field size if the actual outer radius of the network is less than $230 \mathrm{~m}$. In the second

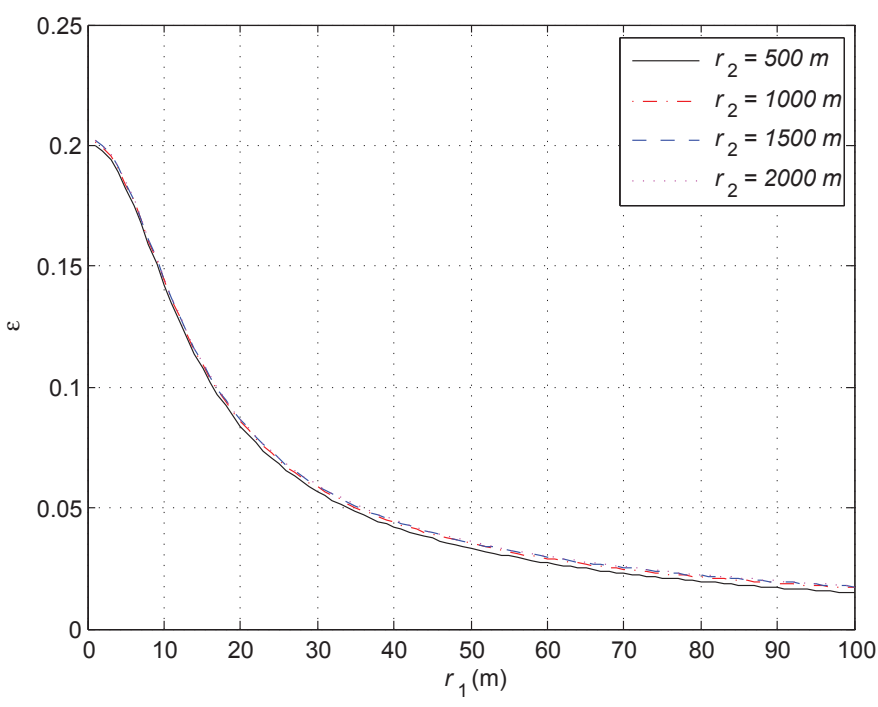

Fig. 4. Outage $\varepsilon$ at the primary receiver vs. inner field radius (i.e., exclusion region) $r_{1}$ for different outer radius values $r_{2}$, with $\lambda=3 \times 10^{-4} / \mathrm{m}^{2}, \theta=2 \pi$, $\alpha=3, \eta=10^{-14} \mathrm{~W}, \rho_{\mathrm{r}}=10^{-6} \mathrm{~W}, \beta=10$, and $\rho=10^{-4} \mathrm{~W}$.

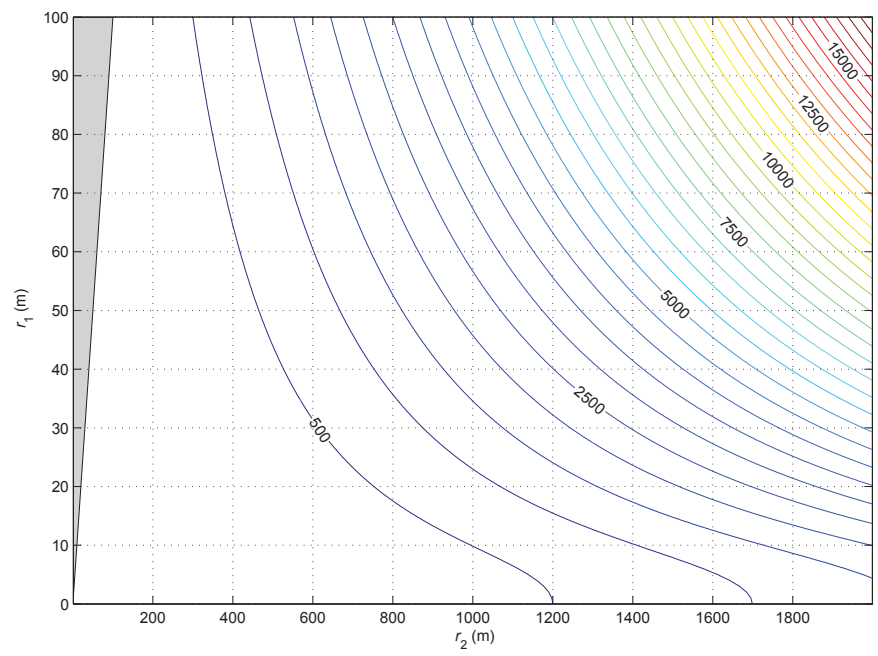

Fig. 5. Maximum average number of the secondary network's transmitting nodes $N_{\max }$ vs. field radii $r_{1}$ and $r_{2}$, with $\varepsilon_{\mathrm{Th}}=0.08, \theta=2 \pi, \alpha=3$, $\rho_{\mathrm{r}}=10^{-6} \mathrm{~W}, \beta=10, \eta=10^{-14} \mathrm{~W}$, and $\rho=10^{-4} \mathrm{~W}$.

case, under the given outage probability constraint, we want to determine the possible spatial deployments of the secondary network to obtain the maximum gains in terms of the average number of secondary transmitters, given by (18).

The derived closed-form expression for the outage probability at the primary receiver due to the finite field secondary network gives the effect of the exclusion region accurately. From Fig. 4, for the same simulation parameters as before, we observe that the inner radius $r_{1}$ has a significant effect on the outage probability at the primary receiver. Even a small change in the inner radius has a much stronger effect on the outage probability than a large change in the outer radius $r_{2}$.

We plot the maximum average number of secondary network transmitter nodes $N_{\max }$ against the field size in Fig. 5, 
using (18) for the same simulation parameters as in the previous cases. The figure shows the contour plot of $N_{\max }$ as a function of field size.

In addition, using (11), it is possible to analyze the effect of other system parameters (power, path loss exponent, etc.) under the given framework.

\section{CONCLUSION}

We derived a closed-form expression for the outage at the primary receiver, with or without an exclusion region, due to a secondary network uniformly distributed over an annular sector. We analyzed the spectrum sharing opportunities under the outage constraint specified by the primary receiver, and we showed them to be significantly dependent on the field size of the secondary network. We analyzed the case where the assumption of an infinite field size can lead to loss in the spectrum sharing opportunity, because the field size is actually finite. We also derived the minimum exclusion radius for which even an infinite field size assumption does not lead to such a loss. Furthermore, we derived the spectrum sharing gains (in terms of the maximum average number of secondary transmitters) for different field sizes. It should be noted that other than networks sharing spectrum, the derived results are applicable to any set of interferers from the same network or other networks around any receiver of study.

\section{ACKNOWLEDGEMENT}

The authors would like to thank Dr. Muhammad Aljuaid for his helpful comments.

\section{REFERENCES}

[1] T. Yucek and H. Arslan, "A survey of spectrum sensing algorithms for cognitive radio applications," IEEE Commun. Surveys Tuts., vol. 11, no. 1, pp. 116-130, 2009.
[2] M. Win, P. Pinto, and L. Shepp, "A mathematical theory of network interference and its applications," Proc. IEEE, vol. 97, pp. 205-230, Feb. 2009.

[3] M. Vu, N. Devroye, and V. Tarokh, "On the primary exclusive region of cognitive networks," IEEE Trans. Wireless Commun., vol. 8, no. 7, pp. 3380-3385, July 2009.

[4] R. Menon, R. Buehrer, and J. Reed, "On the impact of dynamic spectrum sharing techniques on legacy radio systems," IEEE Trans. Wireless Commun., vol. 7, no. 11, pp. 4198-4207, Nov. 2008.

[5] M. Aljuaid and H. Yanikomeroglu, "A cumulant-based investigation of the impact of secondary users' field size on spectrum sharing opportunities," accepted to IEEE Trans. Veh. Technol., May 2011.

[6] A. Ghasemi and E. Sousa, "Interference aggregation in spectrum-sensing cognitive wireless networks," IEEE J. Sel. Topics Signal Process., vol. 2, no. 1, pp. 41-56, Feb. 2008.

[7] R. Dahama, K. Sowerby, and G. Rowe, "Outage probability estimation for licensed systems in the presence of cognitive radio interference," in IEEE Vehicular Technology Conference, Apr. 2009.

[8] M. Timmers, S. Pollin, A. Dejonghe, A. Bahai, L. Van der Perre, and F. Catthoor, "Accumulative interference modeling for cognitive radios with distributed channel access," in International Conference on Cognitive Radio Oriented Wireless Networks and Communications, May 2008.

[9] V. Mordachev and S. Loyka, "On node density - outage probability tradeoff in wireless networks," IEEE J. Sel. Areas Commun., vol. 27, no. 7, pp. 1120-1131, Sept. 2009.

[10] M. Khoshkholgh, K. Navaie, and H. Yanikomeroglu, "Impact of the secondary network on the outage performance of the primary service in spectrum sharing," in IEEE International Conference on Communications, May 2010.

[11] M. Aljuaid and H. Yanikomeroglu, "Identifying boundaries of dominant regions dictating spectrum sharing opportunities for large secondary networks," in IEEE International Symposium on Personal Indoor and Mobile Radio Communications, Sept. 2010.

[12] J. Venkataraman, M. Haenggi, and O. Collins, "Shot noise models for outage and throughput analyses in wireless ad hoc networks," in IEEE Military Communications Conference, Oct. 2006.

[13] J. Kingman, Poisson Processes. Oxford University Press US, 1993.

[14] F. Baccelli, B. Blaszczyszyn, and P. Muhlethaler, "An Aloha protocol for multihop mobile wireless networks," IEEE Trans. Inf. Theory, vol. 52, no. 2, pp. 421-436, Feb. 2006.

[15] I. S. Gradshteyn and I. M. Ryzhik, Tables of Integrals, Series, and Products, 7th ed. Academic Press, 2007. 